\title{
DE OLHO NA INFECÇÃO: narrativa discente sobre o cotidiano do enfermeiro na Comissão de Controle de Infeção Hospitalar (CCIH)
}

\author{
Rosângela Vidal de NEGREIROS ${ }^{1}$ \\ Mikael Lima BRASIL ${ }^{2}$ \\ Javanna Lacerda Gomes da Silva FREITAS ${ }^{2}$ \\ Joseana de Almeida DIAS ${ }^{3}$ \\ Silvana Pontes de OLIVEIRA ${ }^{3}$ \\ Suzana Pereira Cardoso de MEDEIROS ${ }^{3}$
}

${ }^{1}$ Enfermeira, Mestre em enfermagem, docente do Curso de graduação em enfermagem do Centro de Ciências Biológicas e da Saúde (CCBS) da Universidade Federal de Campina Grande (UFCG). E-mail: rosangelavn@ufccg.edu.br

${ }^{2}$ Discente do curso de graduação em enfermagem do CCBS da UFCG;

${ }^{3}$ Enfermeira graduada pelo CCBS da UFCG.

Recebido em: 30/12/2015 - Aprovado em: 15/10/2016 - Disponibilizado em: 18/12/2016

\section{RESUMO:}

No Brasil, tem-se ampliado a vigilância às infecções hospitalares por meio de Comissões de Controle de Infecção Hospitalar (CCIH), as quais contribuem para o monitoramento da qualidade da assistência. Logo, objetiva-se refletir, sob a ótica discente, a atuação do enfermeiro na CCIH a partir da vivência em um Hospital Universitário do Interior nordestino. Estudo de vivência, do tipo relato de experiência, que realizou um resgate narrativo a partir das concepções e reflexões trazidas pelo estágio curricular supervisionado no campo de atuação da enfermagem na CCIH no mês de maio de 2015. A inserção no âmbito da CCIH proporcionou a execução de ações de prevenção, vigilância e controle das infecções hospitalares através da sua busca ativa. Deste modo, o ponto de chegada desta experiência se releva pelo conhecimento adquirido sobre as infecções hospitalares, a importância da CCIH nas instituições e a reflexão sobre o processo de trabalho em enfermagem e saúde para o bom funcionamento das instituições hospitalares e a prestação de um cuidado com maior qualidade. Assim, acredita-se que esta narrativa seja um produto que possa contribuir para reflexões sobre rotinas em $\mathrm{CCIH}$, como também a necessidade do processo de trabalho em saúde como fundamental à prática de enfermagem e, consequentemente, ao o cuidado.

Palavras-chave: Infecção Hospitalar. Enfermagem. Reflexão. Cuidado. Saúde.

\section{KEEP ON EYE ON INFECTION: narrative of students about the nurse daily at the Hospital Infection Control Commission (HICC)}

\begin{abstract}
:
In Brazil, it has been applied the surveillance in hospital infection through Hospital Infection Control Commission (HICC) that contribute to monitoring the quality of the assistance. Thereafter, the aim reflects, based on students vision, the performance of nurse at HICC inside of Alcides Carneiro Academic Hospital in the Northeast of Brazil. Experience study, with the type of experience report it was fulfilled a narrative rescue based on reflections and conceptions brought through the supervised curricular internship inside of nursing acting at HICC in may 2015. The insertion on the scope of HICC provided the accomplishment of action of prevention, surveillance, and control of hospital infections through
\end{abstract}


active search. Thus, the results of this experience was important because of the knowledge that was acquired about hospital infections, the importance of HICC at the institutions and the reflection about the process of nursing work and the health to the proper functioning of hospital institutions and care with quality. Therefore, it is believed that this narrative is a product that can contribute to reflect about the routine of HICC, besides the need of health work process as a principal practice in nursing, and also related to the care.

Keywords: Hospital Infection. Nursing, Reflection. Care. Health.

\section{INTRODUÇÃO}

A rotina hospitalar a partir de internações traz consigo um fator relevante para uma condição imprescindível para um olhar diferenciado no que concerne a qualidade do serviço prestado: a infecção hospitalar (IH).

Assim, compreende-se que Infecção hospitalar é aquela adquirida após admissão do paciente no hospital cuja manifestação pode ter ocorrido durante a internação (72 horas) ou após a alta, podendo ter relação com a hospitalização ou com procedimentos hospitalares. Atualmente, a infecção hospitalar é uma das principais causas de mortalidade nosocomial, podendo estar associada a doenças graves, intervenções médicas e cirúrgicas e complicações a elas relacionadas (OLIVEIRA; KOVNER; SILVA, 2010).

Guimarães (2011) acrescenta que a Infecção Hospitalar se trata de uma relevante causa de morbidade e de mortalidade, destacando-se como um problema de saúde pública.

Também é interessante notar que há uma gradativa apreciação da terminologia Infecção Relacionada à Assistência à Saúde
(IRAS), uma vez que abrange, a partir das informações trazidas por Brasil (2013), não só a infecção adquirida no hospital, mas também aquela relacionada a procedimentos realizados em ambulatório, durante cuidados domiciliares e a infecção ocupacional adquirida por profissionais de saúde (médicos, enfermeiros, fisioterapeutas, entre outros)

Desta maneira, é necessário conhecer com o máximo de rigor a dimensão do problema e obter informações sobre a frequência dessas infecções, e a respectiva gravidade, bem como os fatores de risco associados de forma a estabelecer as respectivas prioridades para a intervenção (PINA et al., 2010).

Considerando o risco significativo à saúde dos usuários dos hospitais e que a sua prevenção e controle envolvem medidas de qualificação da assistência hospitalar, encontra-se na Portaria $n^{\circ} 2616$ de 12 de maio de 1998 a necessidade de um Programa de Controle de Infecções Hospitalares (PCIH) através da constituição da Comissão de Controle de Infecção Hospitalar (CCIH) a qual, preferencialmente, terá como um dos membros executores o enfermeiro (BRASIL, 1988). 
O papel do enfermeiro na temática também é corroborado e mencionado pela Lei que regulamenta o exercício profissional (LEI 7.498/86) quando Brasil (1986) apresenta, entre as funções do enfermeiro, prevenção e controle sistemático da infecção hospitalar.

Assim, ainda destaca-se que a portaria 2616 de 12 de maio de 1998 é composta por cinco anexos com as diretrizes e normas para a prevenção e controle das infecções hospitalares, que discorrem sobre: organização e competências do programa e da comissão de controle de infecção; conceito e critérios diagnósticos das infecções hospitalares; orientações sobre a vigilância epidemiológica das infecções hospitalares e seus indicadores e recomendações sobre a lavagem das mãos e outros temas como uso de germicidas, microbiologia, lavanderia e farmácia, dando ênfase à observância de publicações anteriores do Ministério da Saúde.

Dentre as principais mudanças introduzidas, destaca-se o fato de que ela voltou a preconizar a existência de um grupo executivo, contratado especificamente para as ações de controle de infecção, alterando sua composição em termos quantitativos e qualitativos (CARDOSO; SILVA 2004).

Nessa perspectiva, é compreensível a necessidade da implementação de uma comissão que se dedique à infecção hospitalar, de uma maneira que os agravos à assistência à saúde possam ser minimizados através do caráter de vigilância e prevenção, de uma forma que medidas possam ser adotadas para o controle das infecções hospitalares.

Dessa forma, Brasil (1998) ainda declara, entre outras, como competências da CCIH: elaborar, implementar, manter e avaliar programa de controle de infecção hospitalar, adequado às características e necessidades da instituição, contemplando no mínimo, ações relativas a: Implantação de um Sistema de Vigilância Epidemiológica das Infecções Hospitalares; Adequação, implementação e supervisão das normas e rotinas técnico operacionais, visando à prevenção e controle das infecções hospitalares; Capacitação do quadro de funcionários e profissionais da instituição, no que diz respeito à prevenção e controle das infecções hospitalares; Uso racional de antimicrobianos, germicidas e materiais médico hospitalares; Avaliar, periódica e sistematicamente, as informações providas pelo Sistema de Vigilância Epidemiológica das infecções hospitalares e aprovar as medidas de controle propostas pelos membros executores de $\mathrm{CCIH}$; Realizar investigação epidemiológica de casos e surtos, sempre que indicado, e implantar medidas imediatas de controle entre outros.

Sob essa ótica, observa-se a necessidade da atuação do enfermeiro como 
fundamental na prevenção de infecções como um campo de trabalho imprescindível à prática profissional e a constituição de uma eficaz rotina a ser problematizada pela reflexão.

\section{OBJETIVO}

Refletir, sob a ótica discente, a atuação do enfermeiro na Comissão de Controle de Infecção Hospitalar (CCIH) a partir da vivência em um Hospital Universitário do Interior nordestino.

\section{METODOLOGIA}

\section{Estudo}

exploratório,

de

empírico-descritivo,

experiência vivenciado por discentes do curso de enfermagem da Universidade Federal de Campina Grande, durante o de estagio curricular Supervisionado II, com carga horária de 420 horas. A experiência sucedeu na Comissão de Controle de Infecção Hospitalar (CCIH) do Hospital Universitário Alcides Carneiro, em maio de 2015.

Concorda-se com a apresentação feita por Holliday (2006) quando coloca que a reflexão sistematizadora busca entrar na dinâmica das experiências se encontrando com processos sociais vivos e complexos, percebendo suas relações a partir da própria lógica, absorvendo ensinamentos que possam contribuir tanto com a teoria, quanto com a prática.

Deste modo o relato de experiência propõe tornar visível e compartilhar com outros profissionais e estudantes uma vivência prática, funcionando como um instrumento de pesquisa descritiva que proporciona uma reflexão sobre uma ação ou um conjunto de ações vivenciadas (SANTOS; ALMEIDA; REIS, 2013).

Conforme De Biasi e Pedro (2009), a escolha do relato de experiência possibilita a avaliação de um conjunto de dados, permitindo um aprofundamento sobre o tema, por meio da reflexão sobre as ações desenvolvidas pelo enfermeiro, fundamentada na literatura.

De acordo com a Lei $\mathrm{n}^{\circ} 11.788$, de 25 de Setembro de 2008 (BRASIL, 2008) estágio é um ato supervisionado que se desenvolve no ambiente de trabalho intencionando a preparação para o trabalho produtivo.

Desta maneira, O Estágio Curricular Supervisionado II é uma modalidade de ensino obrigatória no Curso de Graduação em Enfermagem, que tem o propósito de propiciar ao acadêmico do último semestre do curso, uma visão de sua profissão de forma ampla e concreta. Apresenta como objetivo geral: Desenvolver habilidade técnicocientífica para atuar em serviços de saúde integrando os aspectos: planejamento, execução, supervisão, treinamento e avaliação 
das atividades de enfermagem, tomando como referencial teórico os princípios do Sistema Único de Saúde (SUS), a Lei do Exercício Profissional.

Assim, no ECS, o aluno deverá se assumir progressivamente como enfermeiro percebendo com clareza suas limitações e entendendo a necessidade de apoio para a solução dos problemas que envolvem o processo saúde-doença e as suas intervenções. Assim, o aluno estará apto a assegurar um conjunto fundamental de competências que permita a sua inserção nos vários níveis de assistência à saúde. (PROJETO PEDAGÓGICO DO CURSO DE ENFERMGEM - CCBS/UFCG, 2011)

Logo, o delineamento desta experiência se dá a partir das concepções formuladas por CIDAC e Holliday (2007) quando declaram 5 etapas que norteiam a sistematização de experiências:

1) O ponto de partida: a experiência construída a partir das reflexões da prática de enfermagem na Comissão de Controle de Infecção Hospitalar (CCIH);

2) As perguntas iniciais: Como refletir a prática do enfermeiro na CCIH? Que contribuições a atuação do enfermeiro na $\mathrm{CCIH}$ pode trazer a qualidade da assistência?

\section{3) Recuperação do processo vivido:}

Reconstrução do processo vivenciado a partir do pensamento crítico reflexivo;
4) A reflexão de fundo: descrição sintética do processo vivido.

5) Os pontos de chegada: principais conclusões a partir do aprendizado sobre a experiência vivida.

\section{NARRATIVA DISCENTE: a possibilidade de uma reflexão}

Para Ferreira (2010) a assistência à saúde vem evoluindo a partir dos avanços científicos e tecnológicos, refletido em melhoria nas ações de saúde para a população. Porém, percebe-se a persistência de problemas de IH como um grave problema de saúde pública, tanto pela sua abrangência como pelos elevados custos sociais e econômicos. Sendo assim, o risco de se adquirir infecções é determinado pela suscetibilidade do paciente e pelos procedimentos clínicos e invasivos durante a hospitalização.

A importância da enfermagem no controle da IH existe desde que Florence Nightingale sinalizou uma preocupação direcionada a essa problemática e, durante a Guerra da Criméia, padronizou procedimentos de cuidados de enfermagem voltados à higienização e limpeza dos hospitais como a introdução de técnicas de antissepsia (HADDAD; SANTOS, 2011).

Assim, observando o desenvolvimento histórico que relaciona a enfermagem e a IH, 
compreende-se que o seu diagnóstico é de atribuição da $\mathrm{CCIH}$, além de ser responsabilidade dos membros executores realizar a busca ativa de casos.

Dessa forma, a $\mathrm{CCIH}$ atua principalmente com função normativa, ou seja, estabelece normas a serem seguidas pelos profissionais da saúde durante a execução de suas atividades, com finalidade de minimizar os riscos de IH. Além de ser responsável por estabelecer medidas de vigilância; educação permanente dos profissionais e ações educativas que incluem as reuniões com os demais membros da Comissão e o contato diário com os demais profissionais (FERREIRA, 2010).

É seguindo esta ótica que Fonseca e Parcianello (2014) compreendem que a IH é uma das principais causas de morte entre pacientes internados, variando as taxas de mortalidade conforme a etiologia, doença de base, entre outros. Vale salientar a existência de um impacto econômico, uma vez que a IH resulta em novos procedimentos terapêuticos de elevado custo e, consequentemente, prolongamento no tempo de internação.

Logo, a prática de enfermagem na $\mathrm{CCIH}$ vivenciada retoma os seguintes aspectos do processo narrado: a busca ativa de casos, realizado pela equipe da Comissão a partir da revisão dos prontuários e dos exames de culturas dos pacientes. No qual, no prontuário identifica-se a data de admissão, o diagnóstico na internação, e nos resultados de exames laboratoriais avalia-se em especial o que foi avaliado pela microbiologia, além de avaliar leito a leito os sinais e sintomas que possam indicar algum achado de IH. Todos esses procedimentos são importantíssimos para notificações de IH.

Dentre as atividades realizadas pelo enfermeiro, no controle de infecção, destacam-se as seguintes: diagnosticar e notificar os casos de $\mathrm{IH}$; identificar os riscos de infecção; inspecionar a correta aplicação de técnicas assépticas; avaliar e orientar a implantação de medidas de isolamento; utilizar medidas que previnam a disseminação de microorganismos; ser um elo entre os setores do hospital; realizar educação em saúde; executar ações de vigilância sanitária nos setores do hospital e, consequentemente, elaborar medidas preventivas ou corretivas.

Sendo assim, foi perceptível, na prática, a transferência de responsabilidade do controle das IH para a CCIH. Todavia, salientamos que prevenir IH é função e dever de todos os professionais. Desta forma, ressalta-se que os controladores de infecção têm a responsabilidade de regular a política institucional para prevenir e controlar a infecção. Porém, o sucesso das ações dependerá do envolvimento de todos os profissionais que atuam na assistência hospitalar. 
Ainda se menciona, nesta experiência, que muitas dificuldades ainda são enfrentadas pelos profissionais, por questões físicas, emocionais, funcionais e/ou estruturais que interagem na forma de organização da CCIH. Deste modo, verificou-se a discreta incompreensão sobre o papel do enfermeiro da Comissão.

Percorrendo a vivência apresentada, descreve-se as atividades discentes a partir das necessidades levantadas no Estágio Curricular Supervisionado II, tanto em formação teórica quanto prática, que culminaram na inserção no processo de trabalho da $\mathrm{CCIH}$, realizando atividades que fazem parte das normas e rotinas, dentre elas a busca de resultados de culturas laboratoriais para identificação da presença de microrganismos em materiais como sangue, urina, secreções, e lesões de pacientes admitidos no hospital.

Foi realizada a busca ativa em prontuário, e no leito dos usuários das UTI infantil e adulto, Centro Cirúrgico e alas clínicas. Além disso, investigaram-se os antibióticos em uso, como também todo o seu esquema, como também a necessidade em observar a existência de resistência antimicrobiana.

Nas UTI's priorizou-se identificar o risco de infecção relacionada ao uso de sonda vesical de demora, sonda nasogástrica, à ventilação mecânica e ao acesso venoso central. No Centro Cirúrgico foram analisadas, através do livro de registro geral, a quantidade de cirurgias realizadas no mês, bem como sua classificação de acordo com o seu grau de contaminação.

Vivenciando a prática, percebeu-se que o período de formação do enfermeiro não pode estar direcionado somente para o desenvolvimento de ações técnicas previsíveis e definidas. Atuar na $\mathrm{CCIH}$, sem dúvida, se mostra como uma experiência fundamental para a formação do enfermeiro no planejamento de sua assistência. Dessa forma, o que já foi construído poderá ser sedimentado e um novo desafio proposto.

\section{CONSIDERAÇÕES FINAIS}

O estágio curricular supervisionado II é uma etapa extremamente expressiva no processo de formação do aluno, posto que proporciona ao mesmo vivenciar a rotina hospitalar por um período prolongado, como também é uma alternativa fundamental para que haja uma familiaridade maior com as atribuições do profissional de enfermagem do nível superior. Afinal, o discente é inserido nos ambientes hospitalares para que se capacite para a futura atuação profissional.

Atuar na $\mathrm{CCIH}$ foi fundamental para relevar a importância da prática da enfermagem enquanto membro da comissão. Assim, compreende-se que o processo de 
formação acadêmica do enfermeiro deve estar voltado para uma assistência segura e embasada em conhecimentos epidemiológicos e preventivos.

Durante essa vivência, foi imprescindível a inserção dos discentes nas atividades da $\mathrm{CCIH}$, os quais tiveram a oportunidade de participar das ações de vigilância e educação, funcionando, também, como importantes reforços nas medidas de controle das IH através da educação permanente e participação da equipe multiprofissional.

Deste modo, o ponto de chegada desta experiência se releva pelo conhecimento adquirido sobre $\mathrm{IH}$, a importância da $\mathrm{CCIH}$ nas instituições e a reflexão sobre o processo de trabalho em enfermagem e saúde para o bom funcionamento das instituições hospitalares e a prestação de um cuidado com maior qualidade.

Portanto, acredita-se que esta narrativa seja um produto que possa contribuir para reflexões sobre rotinas em $\mathrm{CCIH}$, como também a necessidade do processo de trabalho em saúde como fundamental à prática de enfermagem e, consequentemente, ao o cuidado.

\section{REFERÊNCIAS}

BRASIL. Ministério da Saúde. Agência Nacional de Vigilância Sanitária. Critérios
Diagnósticos de Infecções Relacionadas à Assistência à saúde. Brasília: ANVISA, 2013.

Lei 7.498, de 25 de junho de 1986.

Dispõe sobre a Regulamentação do Exercício da Enfermagem e dá outras providências.

Brasília: Ministério da Saúde; 1986.

Disponível em:<http://www2.camara.gov.br/internet/legis lacao/legin.htm>. Acesso em 20 dez. 2015.

BRASIL. Portaria n ${ }^{\circ}$ 2616/MS/GM, de 12 de maio de 1998. Dispõe sobre o Programa de Controle de Infecção Hospitalar. Diário Oficial [da] República Federativa do Brasil, Brasília, DF, 13 mai 1998. Disponível em: <http://www.anvisa.gov.br/legis/portarias/261 6-98.htm>Acesso em: 18 jun 2015.

\section{LEI N ${ }^{\circ} 11.788$, DE $25 \mathrm{DE}$}

SETEMBRO DE 2008. Dispõe sobre o estágio de estudantes; altera a redação do art. 428 da Consolidação das Leis do Trabalho CLT, aprovada pelo Decreto-Lei no 5.452, de 1o de maio de 1943, e a Lei no 9.394, de 20 de dezembro de 1996; revoga as Leis nos 6.494 , de 7 de dezembro de 1977 , e 8.859 , de 23 de março de 1994, o parágrafo único do art. 82 da Lei no 9.394, de 20 de dezembro de 1996, e o art. 60 da Medida Provisória no 2.164-41, de 24 de agosto de 2001; e dá outras providências. Disponível em: <http://www.planalto.gov.br/ccivil_03/_ato20 07-2010/2008/lei/111788.htm> Acesso em 28 Ago. 2015.

CARDOSO, Renata da Silva; SILVA, Maria Anice da. A percepção dos enfermeiros acerca da comissão de infecção hospitalar: desafios e perspectivas. Texto contexto - enferm., Florianópolis , v. 13, n. spe, p. 50-57, 2004.

CIDAC; HOLLIDAY, Oscar Jara. Sistematização de Experiências: aprender a dialogar com os processos. Rio de Janeiro: CIDAC, 2007.

DELAGEB; Débora Gotardelo Audebert; SILVA Girlene Alves da; Prevenção e 
controle das infecções hospitalares: um desafio em instituições de saúde de juiz de fora. Revista Baiana de Saúde Pública. v.35, n.4, p.984-1000 out./dez. 2011.

DE BIASI, Luciana Spinato; PEDRO, Eva Neri Rubim. Vivências de aprendizagem do cuidado na formação da enfermeira. Rev. esc. enferm. USP, São Paulo, v. 43, n. 3, set. 2009.

FONSECA, Graziele Gorete Portella; PARCIANELLO, Márcio Kist. O enfermeiro na comissão de controle de infecção hospitalar na perspectiva ecossistêmica: relato de experiência. R. Enferm. Cent. O. Min. v. 4. n. 2, Maio/agosto, 2014.

FERREIRA, Rodrigo Silva. Atuação da comissão de controle infecção hospitalar (CCIH) na redução da infecção: Um estudo no Hospital da Criança Santo Antônio. Norte Científico. Roraima, v.5, n.1, dezembro de 2010.

GUIMARAES, Aline Caixeta et al . Óbitos associados à infecção hospitalar, ocorridos em um hospital geral de Sumaré-SP, Brasil. Rev. bras. enferm., Brasília , v. 64, n. 5, p. 864869 , Oct. 2011

HADDAD, Veronica Cristin do Nascimento; SANTOS, Tânia Cristina Franco. A teoria ambientalista de florence nightingale no ensino da escola de enfermagem Anna Nery (1962 - 1968). Esc. Anna Nery, Rio de Janeiro , v. 15, n. 4, p. 755-761, Dec. 2011.

HOLLIDAY, Oscar Jara. Para sistematizar experiências. tradução de: Maria Viviana V. Resende. 2. ed., revista. Brasília: MMA; 2006.

OLIVEIRA, Adriana Cristina de; KOVNER, Christine Tassone; SILVA, Rafael Souza da. Infecção hospitalar em unidade de tratamento intensivo de um hospital universitário brasileiro. Rev. Latino-Am. Enfermagem, Belo Horizonte, MG, 18(2), mar-abr 2010.

PINA, Elaine et al . Infecção relacionada com a prestação de cuidados de saúde: infecções da corrente sanguínea (septicemia). Rev. Port. Sau. Pub. Lisboa, v. 28, n. 1, 2010.

\section{PROJETO PEDAGÓGICO DO CURSO DE ENFERMAGEM DO CCBS-UFCG. CCBS: UFCG, 2011.}

SANTOS, Debora de Souza; ALMEIDA, Lenira Maria Wanderley Santos de; REIS, Renata Karina. Programa de Educacao pelo Trabalho para Saude: experiencia de transformacao do ensino e pratica de enfermagem. Rev. esc. enferm. USP. São Paulo, v. 47, n. 6, Dec. 2013. 